\title{
Experimental Analysis on Incompressible circular and non- circular Fluid Jet through Passive Control Method
}

\author{
Laxmireddy Peram ${ }^{1}$, Bhaskar Bommera ${ }^{2}$ \\ ${ }^{1,2}$ Mechanical Engineering, Geethanjali College of Engineering and Technology, Cheeryal(V), Keesara(M), \\ Medchel Dist., Telangana, India
}

\begin{abstract}
Fuel mixing, cooling system and chemical mixing is very predominant in automobile industries, pharmaceutical laboratories etc. for the better performance. In an effort to increase mixing tendency, we are planning to control the incompressible fluid flow jets by a passive control method, using vortex generators like Non-Circular cross sections in this paper. The experimental setup with non-circular jet flow and compare with a circular jet flow. Here different geometrical shapes with equivalent area are taken to study the flow. Rectangular models with different aspect ratios studied are $L / D=1$ and 2 . And these can be compared with the circular orifice of $10 \mathrm{~mm}$ diameter. Rectangular model shows significant effect on mixing mechanism.
\end{abstract}

Keywords: passive control method, noncircular jet, aspect ratio, circular orifice, mixing mechanism

\section{Introduction}

Noncircular jets have been the topic of extensive research in the aerospace; it has the ability to enhance the mixing characteristics of a jet, which will greatly improve the performance of system. Non circular jets have potential to entrain ambient fluid more effectively than comparable circular jets.

\section{Characteristics of Jet Flow With Vortices}

Large-scale coherent structures control the dynamics of all free shear flows and wakes. These twodimensional structures were found to play an important role in entrainment and mixing processes in incompressible shear layers ${ }^{1}$. The formation of coherent structures in a shear layer is initiated by KelvinHelmholtz instability and the behavior of fully turbulent shear layers suggest that large eddies constitute the dominant instability of the flow governed by Rayleigh's equation for inviscid flows. The exponential growth of the velocity and vorticity perturbations leads to a nonlinear process that eventually causes the roll-up of the shear layer vortices ${ }^{2,3}$.

The initial vortices grow in the shear layer and coalesce as they are convected downstream in a "pairing" process ${ }^{4}$. Due to merging and entrainment, the shear layer spreads, and the frequency associated with the large vortices decreases. The irrotational entrainment by the large-scale structures leaves the entrained fluids essentially unmixed during the lifetime of the vortices. Nonetheless, intense mixing occurs during pairing or other amalgamation processes. Some distance downstream of the splitter plate trailing edge, a secondary, span wise instability appears, leading to the development of stream wise vortices 5 .

In the shear layer of a jet, the physical dimensions of the nozzle introduce new length scales, not present in a plane shear layer. The number of vortex interactions is limited by the distance between the nozzle and the location where the shear layer surrounding the potential core merges. The jet flow characteristics at the end of the potential core are determined by the jet-column instability ${ }^{6}$.

\section{Jet Control}

Passive control, which uses geometrical modifications of the element from which flow separation occurs to change the shear layer stability characteristics. Some examples of these modifications are: co-flow jets; trip wires in plane shear layers; convoluted splitter plates; non-circular jets such as square jets, and elliptic jets.

\section{Noncircular Jet Characteristics}

Rectangular jets are popular among jet research community owing to their wide practical applications. Rectangular jets find application in fluidics, ink-jet printing, V/STOL aircraft, military aircraft etc. They are also currently in use on stealthy aircrafts and with other rapid mixing technologies ${ }^{7}$. Rectangular jets combine the aspect ratio features of an elliptic jet with corner (vertex) features of square jets. Nozzle exit shape, aspect ratio, initial turbulence level, and Reynolds number affect the development of the jet ${ }^{8}$. The flow field of the rectangular jet may be subdivided into three main regions: potential core region, followed by characteristic International Conference on Recent Innovations in Civil \& Mechanical Engineering $\quad 15 \mid$ Page [i-CAM2K16] DOI: 10.9790/1684-16053021521 
decay region and axisymmetric decay region. In the characteristic decay region, the axial velocity decay is dependent upon orifice configuration and the velocity profiles in the plane of the minor axis of the orifice are found to be similar whereas those in the plane of the major axis plane are non-similar. Three-dimensional turbulent rectangular jets. It has been suggested that, the anomalous behavior of the flow in the two-dimensional region of the jet is associated with the three-dimensional effects ${ }^{9,10}$.

The nozzle geometry and the aspect ratio play an important role on the jet development through these three-dimensional effects. The spreading rate of a rectangular jet is higher at the wide section than the narrow side. This results in axis-switching. The half-width of the jet varies linearly with downstream distance with different slopes for different aspect ratios and initial geometries ${ }^{11}$. The 1distance of the cross-over location from the nozzle was found to be directly proportional to the nozzle aspect ratio ${ }^{12}$. For the contoured rectangular nozzle of aspect ratio 2, the deformation of the rhombus-like jet spread occurs all over the mixing layer. For rectangular slot jets, near-field mixing increases with slot aspect ratio. The jet growth rate in minor axis plane was higher; the jet width in the major axis plane was initially reduced due to the vena contracts effect. Following axis switching, the major axis growth rate became higher than the minor axis, resulting in a second axis switching farther downstream ${ }^{13,14}$.

\section{Present Study}

Present experimental study done on Non circular orifices having aspect ratio 1, 2 and compare with circular orifice of equivalent area. Previous study focus only compressible medium and air only, here we taken water as incompressible medium done experiments at two different heads. Tabulated the center line pressure distribution and transverse pressure distribution. And also locate the axis switching point, centerline decay and jet fall of point.

\subsection{Experimental Facility}

The experimental set up is shown in the Fig 3.1 and the three standard heads are shown in the Fig 3.2.

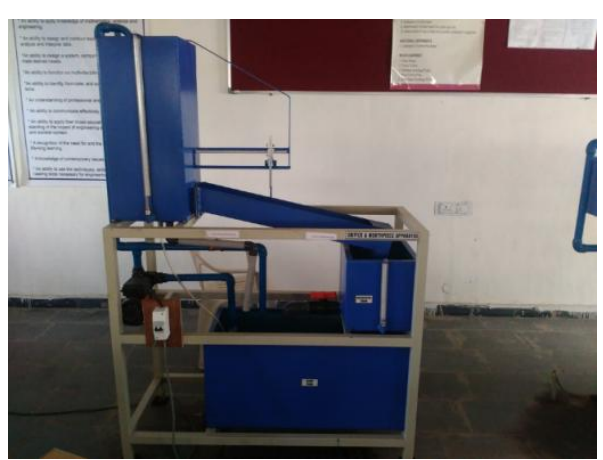

Figure 5.1.1. Orifice mouth piece setup

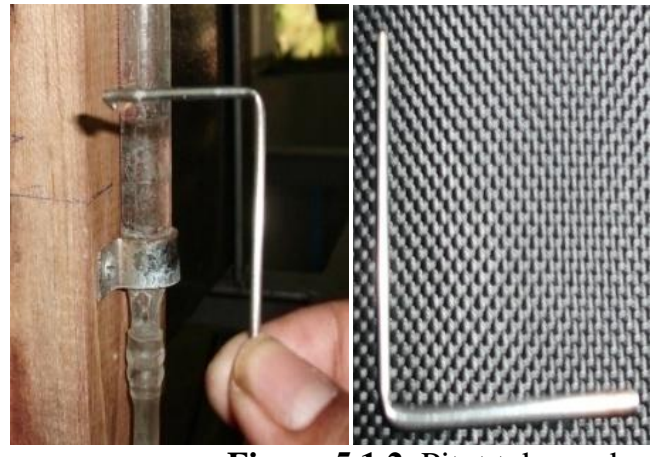

Figure 5.1.2. Pitot tube probe

Here the orifices were made according to the dimensions as follows in the TABLE I. The settling chamber total pressure which was the controlling parameter in this investigation was maintained constant during a run by controlling the head regulating valve. The stagnation pressure leveling the settling chamber gives the different pressure ratios, defined as the ratio of stagnation pressure to the back pressure required for any study. The settling chamber temperature is the same as the ambient temperature and the back pressure is the ambient pressure into which the water jets were discharged. The ambient temperature of the room was almost constant within $0.5^{\circ} \mathrm{C}$ during one experimental run. The stagnation pressure was maintained with an accuracy of $0.1 \%$. During the experimental runs, the settling chamber pressure was assumed to be constant.

This set up consists also a scale to calculate the axial length and also has a radial scales which were fixed to a stand at a distance of $10 \mathrm{~cm}$ in the radial direction of the flow. These scales give the exact length at which the width of the flow can be calculated.

\subsubsection{Instrumentation for Pressure Measurement}

Head at different point can be used to calculate the pressures at that respective point. For calculating the heads that is pressures at different points a simple pitot tube was made and connected to the set up. A burette was fixed to the stand in the radial direction and the pitot probe was connected to the burette by using the rubber tube. The pitot probe was constructed just by taking the needle of the animal vaccination and it was bent into the right angled and the needle diameter is $2 \mathrm{~mm}$ is used and length of $80 \mathrm{~mm}$ is used, which was made of stainless 
steel. As we measure pressure profiles at every location such that probe moves in three dimensionally. So we arrange a wooden stand, in which probe moves in three dimensional ways.

\subsubsection{Experimental Model}

The flow had studied for different shapes of rectangle with aspect ratio of 1 and 2 and circular shape with equivalent area. Rectangular models different aspect ratios $(\mathrm{L} / \mathrm{D}=1,2$ i.e. $8.8 \times 8.8 .6 .3 \times 12.5)$, these can be compared with the circular orifice of $10 \mathrm{~mm}$ dia. In addition to this orifices are placed in diffused mixed burners to study the effect of flame exit of the noncircular orifices.

Experiments were carried out using rectangular, square and circle orifices. Artistic views of the orifices are shown in figures 5.2.1 and 5.2.2 \& figure 5.2.3.

TABLE I: Geometrical Parameters of Incompressible Models

\begin{tabular}{|c|c|c|}
\hline Geometry & Dimensions $(\mathrm{mm})$ & Area $\left(\mathrm{mm}^{2}\right)$ \\
\hline Circle & Diameter $=10$ & 78.53 \\
\hline Rectangle & Length $=12.53$, Breadth $=6.27$ & 78.53 \\
\hline Square & Side $=8.87$ & 78.53 \\
\hline
\end{tabular}
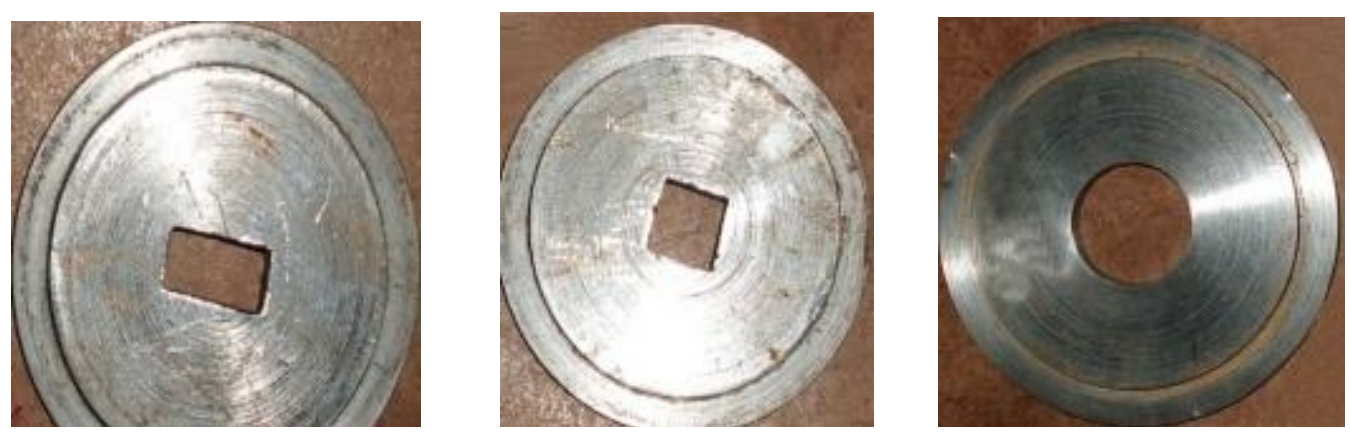

Figure: 5.2.1 Figure: 5.2.2 Figure: 5.2.3

\section{Results And Discussion}

To characterize the water jet characteristics, the primary study is carried out for circular orifice. To understand the nature of propagation of jet, the basic measurement done is centerline pressure measurement. The pressure head measurement is done with Pitot tube which measures total or stagnation pressure head. The measured pressures are gauge pressures, which are to be converted into absolute pressures by adding atmospheric pressure. The measured pressure head is Non-dimensionalised with the pressure head maintained in the tank.

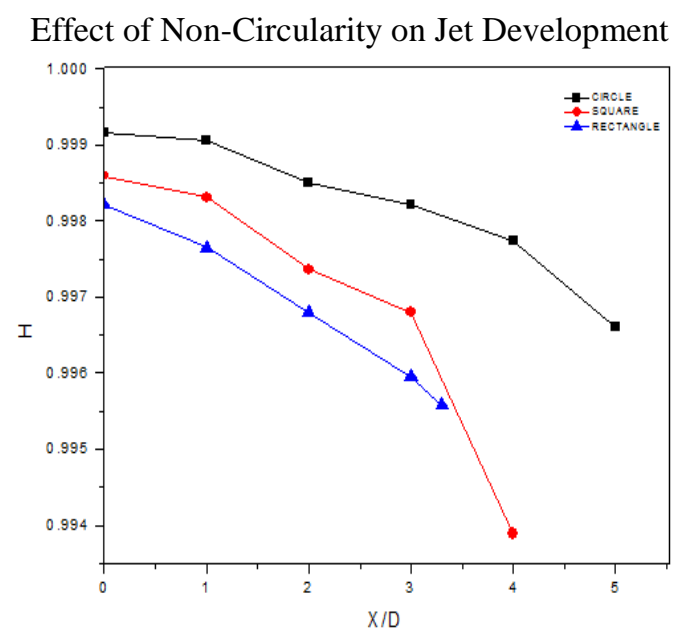

Figure 6.1.1 Centerline pressure distribution at 30 Head (Head Vs Length - diameter ratio) 


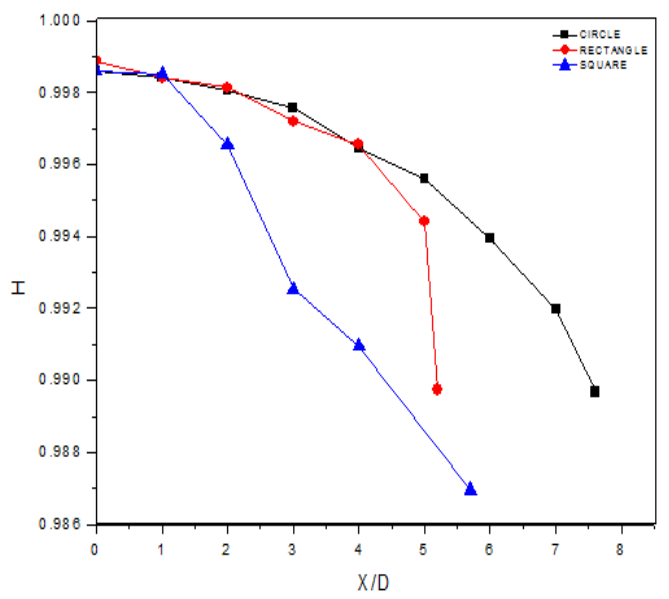

Figure 6.1.2 Centerline pressure distribution at $40 \mathrm{Head}$ (Head Vs Length - diameter ratio)

Fig. 6.1.1 presents the centerline distribution of rectangular and square model along with equal area of circular model at head of $30 \mathrm{~cm}$ of $\mathrm{H}_{2} \mathrm{O}$ and demonstrates the variation of centerline pressure with change of geometry. For this at a particular head, effect of geometry modification is significant for rectangular and square model compared to circle. Rectangular model shows more significant centerline pressure drop compared to other two models.

Figure 6.1.2 presents the centerline distribution of rectangular and square model along with equal area of circular model at head of $40 \mathrm{~cm}$ of $\mathrm{H}_{2} \mathrm{O}$ and demonstrates the variation of centerline pressure with change of geometry. For this at a particular head, effect of geometry modification is significant for rectangular and square model compared to circle. Square model shows more significant centerline pressure drop compared to other two models.

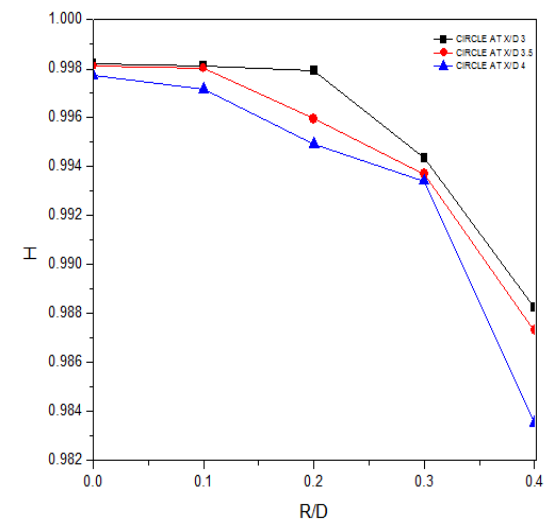

Figure 6.1.3 Radial pressure distribution of Circular Orifice at 30 head (Head Vs transverse length - diameter ratio)

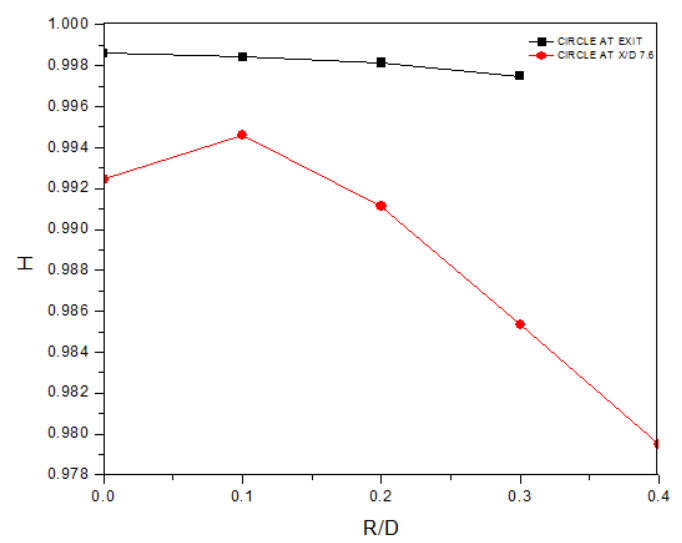

Figure 6.1.4 Radial pressure distribution of Circular orifice at 40 head (Head Vs transverse length - diameter ratio) 
Figure 6.1.3 presents the radial pressure distribution of circular model at head of $30 \mathrm{~cm} \mathrm{of} \mathrm{H}_{2} \mathrm{O}$. To understand the behavior of jet in radial direction, radial pressure distribution is carried out at X/D 3, 3.5, 4. The reason for choosing these axial locations is to understand the pressure distribution before the significant fall of the jet and after the significant fall of the jet. From constant observations, $X / D=3.5$ is identified as jet fall point. Pressure drops significant thereafter. From Fig 4.13, it is observed that pressure drop is significant compared to jet position at before fall of point.

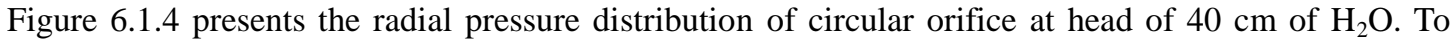
understand the behavior of jet in radial direction, radial pressure distribution is carried out at exit of orifice and jet fall of point. Jet drops at axial location of $X / D=7.6$. There is significant pressure drop at jet fall of point.

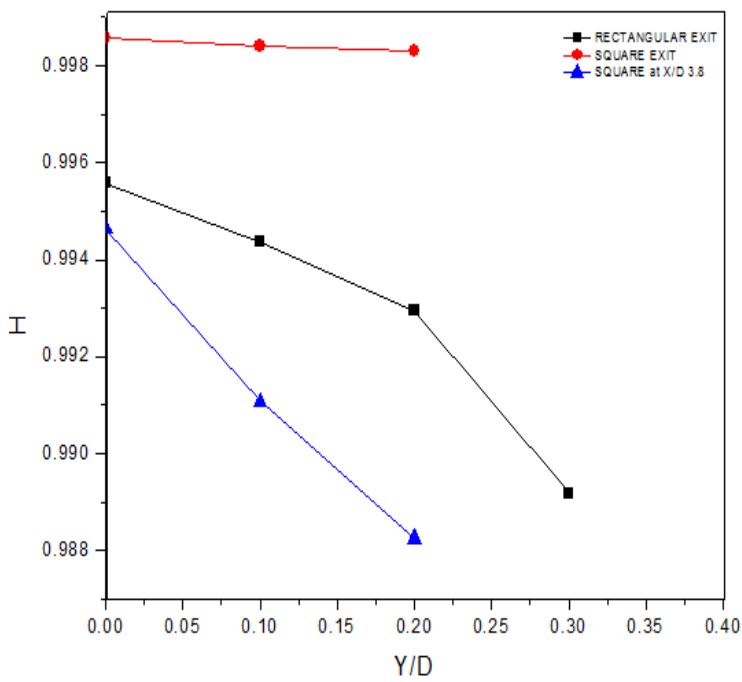

Figure 6.1.5 Pressure distribution along $\mathrm{Y}$ direction for Square and rectangular models at $30 \mathrm{~cm}^{\circ} \mathrm{H}_{2} \mathrm{O}$

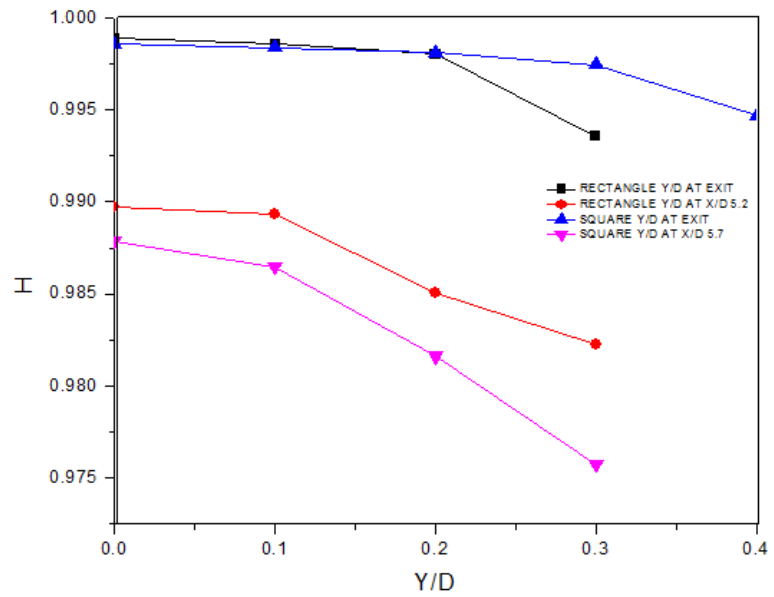

Figure 6.1.6 Pressure distribution along $\mathrm{Y}$ direction for Square and rectangular model at $40 \mathrm{~cm}^{\circ} \mathrm{H}_{2} \mathrm{O}$

Fig. 6.1.5 presents the pressure distribution along $\mathrm{Y}$ direction at head of $30 \mathrm{~cm}$ of $\mathrm{H}_{2} \mathrm{O}$. Pressure distribution taken at exit of rectangular and square orifices and square jet fall of location and clearly demonstrates the pressure variation of the square and rectangular orifice at exit. Rectangular jet has significant pressure drop compared to square jet. At jet fall of location, pressure drop is significant for square jet compared to square jet at exit of orifice.

Fig. 6.1.6 presents distribution along Y direction for square and Rectangular models at head of $40 \mathrm{~cm}$ of $\mathrm{H}_{2} \mathrm{O}$. Pressure is measured at exit of orifice and jet fall of locations and clearly demonstrates the geometrical variation and axial location variation on jet characteristics. The effect of geometrical variation is very minimum at exit. At the head of $40 \mathrm{~cm}$ of $\mathrm{H}_{2} \mathrm{O}$ jet drops for rectangular model at $\mathrm{X} / \mathrm{D}=5.2$ and for square model at X/D = 5.7. Pressure variation is significant for both the models compared to jet location at exit. 


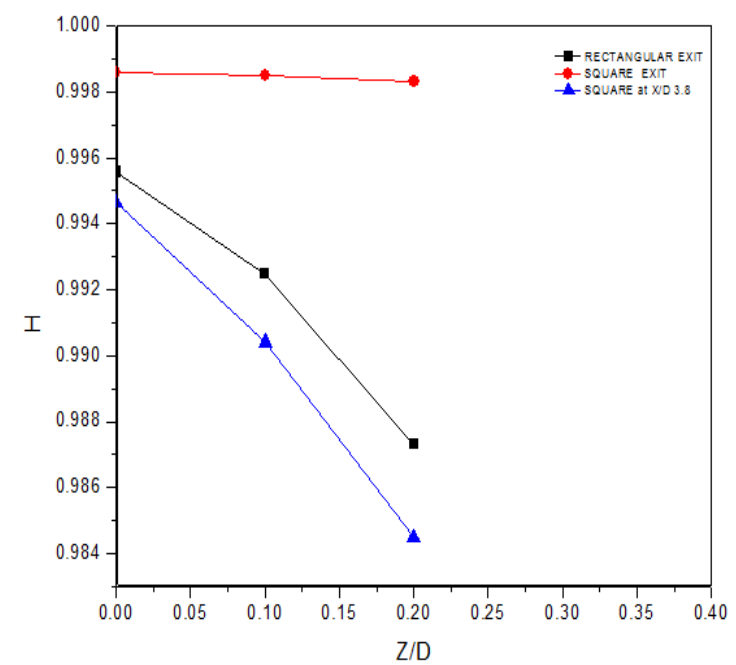

Figure 6.1.7 Pressure distribution along $\mathrm{Z}$ direction for Square and rectangular models at $30 \mathrm{~cm}$ of $\mathrm{H}_{2} \mathrm{O}$

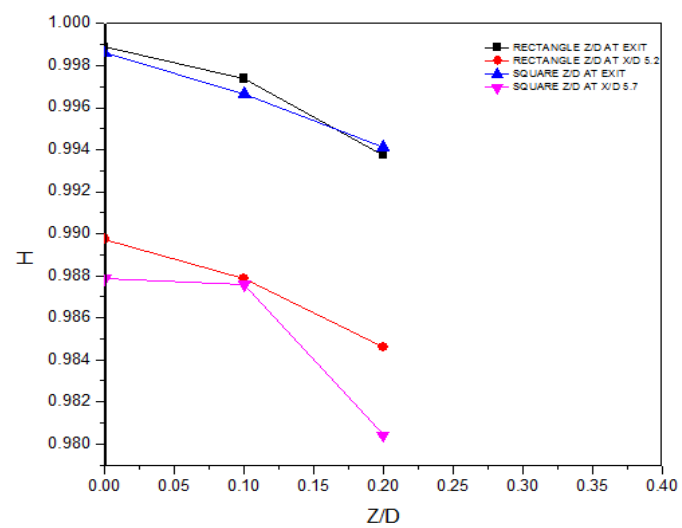

Figure 6.1.8 Pressure distribution along $\mathrm{Z}$ direction for Square and rectangular model at $40 \mathrm{~cm}^{\circ} \mathrm{H}_{2} \mathrm{O}$

Fig. 6.1.7 presents the pressure distribution along $\mathrm{Z}$ direction for square and rectangular models at head of $30 \mathrm{~cm}$ of $\mathrm{H}_{2} \mathrm{O}$. Pressures are measured at exit of rectangular, square models and square jet fall of location and clearly demonstrate the effect of geometrical variation on jet characteristics. Rectangular jet has significant pressure drop compared to square jet at exit of the orifice. Pressure drop is significant for square model at jet fall of location compared exit of orifice.

Fig. 6.1.8 presents the pressure distribution along $\mathrm{Z}$ direction for square and rectangular models at head of $40 \mathrm{~cm}$ of $\mathrm{H}_{2} \mathrm{O}$. Pressures are measured for rectangular, square models at exit and jet fall of locations and clearly demonstrate the geometrical variation and axial location on jet characteristics. The effect of geometrical variation at exit of orifice is very minimum. Rectangular jet falls at $\mathrm{X} / \mathrm{D}=5.2$ and square jet falls at $\mathrm{X} / \mathrm{D}=5.7$. Pressure drop is significant at jet fall of location compared to exit of orifice for both models.

\section{Conclusion}

Effect of geometrical modification is significant for rectangular and square model compared to circle at both the pressure heads. Rectangular jet has significant pressure drop compared to square jet at head of $30 \mathrm{~cm}$ of $\mathrm{H}_{2} \mathrm{O}$. Square model shows more significant centerline pressure drop compared to other two models at head of 40 $\mathrm{cm}$ of $\mathrm{H}_{2} \mathrm{O}$. The effect of geometrical variation is very minimum at exit for the pressure head of $40 \mathrm{~cm}$ of $\mathrm{H}_{2} \mathrm{O}$. The effect of geometrical variation is very significant at exit for the pressure head of $30 \mathrm{~cm}$ of $\mathrm{H}_{2} \mathrm{O}$. Pressure variation is significant for both the models compared to jet location at exit.

\section{References}

[1]. Brown, G. L., and Roshko, A., "On Density Effects and Large Structure in Turbulent Mixing Layers", journal of fluid mechanics, vol.64, 1974 pp.775-816.

[2]. Ho C.M., And Huerre, P., "Perturbed Free Shear Layers", Annual Review Of Fluid Mechanics, Vol.16, Pp. 365-424.

[3]. Morris, P.J., Giridharan, M.G., And Lilley, G.M., "On The Turbulent Mixing Of Compressible Free Shear Layers", Proceedings Of The Royal Society Of London, A 431, 1990 Pp. 219-243. 
Experimental Analysis on Incompressible circular and non-circular Fluid Jet through Passive ..

[4]. Winant, C.D., And Browand, F.K., "Vortex Pairing: The Mechanism Of Turbulent Mixing-Layer Growth At Moderate Reynolds Numbers", Journal Of Fluid Mechanics, Vol. 63, No.2, 1974, Pp. 237-255.

[5]. Dimotakis, P.E., And Brown, G.J., "The Mixing Layer At High Reynolds Number: Large Structure Dynamics And Entrainment", Journal Of Fluid Mechanics, Vol. 78, 1976 Pp. 535-560.

[6]. Bernal, L., And Roshko, A., "Stream Wise Vortex Structures In Plane Mixing Layers", Journal Of Fluid Mechanics, Vol. 170, 1986, Pp. 499-525.

[7]. Knowles, K., And Saddington, A.J. "A Review Of Jet Mixing Enhancement For Aircraft Propulsion Applications" Proceedings Of Imeche. Part G: Journal Of Aerospace Engineering, Vol. 220, 2006, Pp.103-127.

[8]. Sforza, P.M., Steiger, M.H., And Trentacoste, N. "Studies On Three-Dimensional Viscous Jets" Aiaa. J., Vol. 4, 1966, 800-806.

[9]. Nicholas Trentacoste., And Pasquale Sforza "Further Experimental Results For Three-Dimensional Free Jets" Aiaa. J., Vol. 5, No. 5, May 1967, Pp. 885-891.

[10]. Sfeir, A.A. "Investigation Of Three-Dimensional Turbulent Rectangular Jets" Aiaa. J., Vol. 17, No. 10, October 1979, Pp. 1055-1060.

[11]. Krothapalli, A., Bagano D., And Karamachetti, K. “On The Mixing Of Rectangular Jets” Journal Of Fluid Mechanics, Vol. 107, 1981, Pp. 201-220.

[12]. Gutmark, E.J., And Grinstein, F.F. "Flow Control With Noncircular Jets" Annual Review Of Fluid Mechanics, Vol. 31, 1999, Pp. 239-272.

[13]. Tsuchiya Y., Horikoshi C., And Sato, T. “On The Spread Of Rectangular Jets” Experiments In Fluids, Vol. 4, 1986, Pp. 197-204.

[14]. Quinn W.R. "Turbulent Free Jet Flows Issuing From Sharp-Edged Rectangular Slots: The Influence Of Slot Aspect Ratio" Experimental Thermal And Fluid Sciences, Vol.5, 1992b Pp. 203-215. 